\title{
Effectiveness of a Feed Supplement in Advanced Stages of Feline Chronic Kidney Disease
}

\author{
Diana Vergnano', Emanuela Valle', Natascia Bruni ${ }^{2}$, Rita Rizzi³ ${ }^{3}$ Mauro Bigliati ${ }^{2}$ Tiziana Cocca ${ }^{4}$
}

\begin{abstract}
Background: Chronic kidney disease (CKD) is a very common pathology in cats, especially in the geriatric age. A proper renal diet is considered the current standard of care to enhance patients' long-term survival and quality of life. However, when diet alone is not sufficient, it is necessary to supplement it with specific substances: these are phosphate binders and alkalinizing agents. The aim of this study was to evaluate the effectiveness of a feed supplement containing calcium carbonate, calcium lactate gluconate, chitosan and sodium bicarbonate in controlling hyperphosphatemia and metabolic acidosis in cats with severe CKD (IRIS, International Renal Interest Society, stage 3 and 4).

Materials, Methods \& Results: 10 cats (median BW 4.00 (3.20; 5.70) Kg, BCS 3/5 (2.25; 3.75), 11 (8.25;12.00) years) fed with a balanced renal diet were included in the study. To be enrolled in the study cats had to be affected by CKD in stages 3 or 4 and show hyperphosphatemia. Treatment consisted in oral administration of the product (Renal, Candioli Pharma) at $0.2 \mathrm{~g} / \mathrm{kg} / \mathrm{day}$ mixed with the food for 60 days. The animals were evaluated at the beginning of the study and at 15, 30, 60 days (T0, T15, T30, T60) for: clinical condition, BW, BCS, blood pressure and for routinely hematochemical, biochemical and urinary parameters. Owners were asked to assess appetite of the cat, palatability of the supplement, presence of vomit and/or diarrhoea, general health and vitality. All statistical analyses were performed using SAS software. After checking normality data were analyzed using Kruskal-Wallis and Wilcoxon tests. Results are expressed as median (interquartile range). For the parameters $P(P<0.0001), \mathrm{iCa}(P=0.0008)$ and $\mathrm{HCO} 3(P=0.0002)$ there were statistically significant differences among times of supplementation (T0, T15, T30, T60). Statistically significant reduction of serum phosphorus concentration was obtained through the study (reduction of 59\% at T60 vs T0). Also a statistically significant increase of bicarbonate was seen (7\% from T0 to T60). At T60 was also recorded an increase of ionized calcium level, which however was in normal range. For the other laboratory parameters, no statistical difference was recorded. All the owners reported a good palatability of the product. The decrease of vomit and diarrhea episodes and the increase of the appetite reported were statistically significant $(P<0.05)$.

Discussion: The restriction of available dietary phosphorus is now recognised as one of the major contributors in slowing the disease progression and improving survival rates. Phosphate binders are able to absorb phosphate $(\mathrm{P})$ in the intestine, forming insoluble products that are eliminated with the faeces, thus decreasing serum phosphate levels. The phosphate binders contained in the product tested in the present trial were chitosan, calcium lactate gluconate and calcium carbonate. During the study $\mathrm{P}$ decreased significantly from $\mathrm{T} 0$ to $\mathrm{T} 60$, reaching the target post-treatment plasma $\mathrm{P}$ concentration for IRIS stage 3 after 30 days. Another important recommendation for CKD treatment is to use alkalinisation therapy if metabolic acidosis is present. The feed supplement tested in this study also contained sodium bicarbonate. In our study, $90 \%$ of the patients at the inclusion examination had metabolic acidosis. At the end of the study, the median blood bicarbonate concentration was in the normal range, thus reaching the IRIS treatment target. The feed supplement tested was therefore effective in reducing blood phosphate levels and in increasing blood bicarbonate levels, thus improving the cats' clinical conditions for the duration of the study without any adverse effect.
\end{abstract}

Keywords: cats, chronic kidney disease, hyperphosphatemia, metabolic acidosis, feed supplement, phosphate binder. 


\section{INTRODUCTION}

Chronic kidney disease (CKD) is a very common pathology in old cats (more than 15 years; [13]), and it can even affect the $50 \%$ of them [11]. CKD is defined as a kidney disease that has been present for three months or longer [25]. Dogs and cats with CKD are staged according to guidelines developed by the International Renal Interest Society (IRIS) [18].

There is general agreement regarding the nutritional management of CKD when overt signs exist, which includes measures to reduce signs of uremia and to slow down the progression to later stages of disease [12]. Based on clinical trial findings, a renal diet is the therapeutic intervention most likely to enhance longterm survival and quality of life for patients with IRIS CKD stages 3 and 4; as a consequence, diet should be considered as the current standard for care $[2,6,15]$.

When diet alone is not sufficient, CKD cats need to have their diet supplemented with phosphate binders and alkalinizing agents [26]. The aim of this study was to evaluate the effectiveness of a feed supplement containing calcium carbonate, calcium lactate gluconate, sodium bicarbonate and chitosan in cats with CKD in IRIS stages 3 and 4. Since CKD cats often show poor compliance [2], palatability and ease of administration of the product were also evaluated.

\section{MATERIALS AND METHODS}

The study was performed at the Veterinary Clinic Napolivet, in Naples (Italy). An owners' written consent was obtained and all the samples were collected by the same clinician.

Animals

Using the available detailed database of the clinic that included history, physical examination, hematology, biochemistry, urinalysis, and urine protein/ creatinine ratio of the patients, ten cats were selected. Cats were excluded if they were affected by other concomitant diseases like acute kidney injury, prerenal or postrenal azotemia, genitourinary tract inflammation or infection, urinary tract obstruction, chronic heart failure, neoplasia, hyperthyroidism, diabetes.

To be enrolled in the study cats had to be affected by CKD in stages 3 or 4 according to the International Renal Interest Society (IRIS) system [18] (serum creatinine concentration above the reference value, 2.9 $\mathrm{mg} / \mathrm{dL}$, on at least 2 different consecutive occasions) and show clinical signs of polyuria and polydipsia. For the enrollment in the study the animals should also have hyperphosphatemia, i.e. a plasma phosphate concentration higher than $5 \mathrm{mg} / \mathrm{dL}(1.6 \mathrm{mmol} / \mathrm{L})$ for cats in IRIS stage 3 and higher than $6 \mathrm{mg} / \mathrm{dL}(1.9 \mathrm{mmol} / \mathrm{L})$ for cats in IRIS stage 4.

All the animals were fed with the same renal diet (Royal Canin Renal Feline) ${ }^{1}$ from at least eight weeks before the inclusion in the study. The amounts offered were based on the estimated requirements according to FEDIAF Nutritional Guidelines [10]. The same diet had to be maintained for the entire duration of the study. After the enrollment (T0), to the diet was added a feed supplement (Renal) ${ }^{2}$ especially designed for cats with CKD (Table 1). The dosage was $0.2 \mathrm{~g} / \mathrm{kg}$ body weight/day divided in two daily administrations mixed with the feed. The supplement was given for 60 days and analysis were performed at 15 (T15), 30 (T30) and 60 (T60) days.

Table 1. Composition of the feed supplement (Renal).

\begin{tabular}{cc}
\hline Analytical constituents & $\%$ \\
\hline Crude ash & 28 \\
Crude protein & 6.5 \\
Crude fiber & 1 \\
Potassium & 0.02 \\
Calcium & 13.68 \\
Phosphorus & $<0.01$ \\
Sodium & 0.3 \\
\hline
\end{tabular}

Data are expressed as \% on dry matter.

\section{Blood Sampling}

Blood samples were collected from the jugular vein after an overnight fasting and divided into three tubes: a tube without anticoagulant for serum, a K3-EDTA anticoagulant tube for entire blood, and a heparinized tube for hemogas test. Venous haemogas analysis was performed immediately after sampling with a standard analytical device (Vet Scan i-STAT Handheld Analyser) ${ }^{3}$ to assess bicarbonate (HCO3) and ionized calcium (iCA). For complete blood count. $(\mathrm{CBC})$ a standard analytical device (scil Vet $\mathrm{ABC} \mathrm{CM}^{4}$ for the determination was used to evaluate: hematocrit (HT), hemoglobin (HG), red blood cells (RBC), white blood cells (WBC), neutrophils (N), eosinophils (EO), lymphocytes (LYM). For the biochemical analysis an automated analyser (Echo) ${ }^{5}$ was used to obtain creati- 
nine (CREA), blood urea nitrogen (BUN), phosphorus (P), total proteins (TP), albumins (ALB), albumin/ globulin (A/G) ratio, glucose (GLU), alanine transaminases (ALT) alanine aminotransferases (AST), alkaline phosphatases (ALP), bilirubin (BIL) and cholesterol (CHOL) values.

\section{Urine Sampling}

Urinary samples were collected by free catch. Urinalysis included specific gravity (SG) by refractometry and sediment examination. Urine was centrifuged ( 2 min at $1500 \times \mathrm{g}$ ) and supernatant was removed and stored at $+4^{\circ} \mathrm{C}$ to evaluate urine protein (UP) concentration (pirogallol red method) and creatinine concentration (Jaffè method) within $12 \mathrm{~h}$. Urine protein/ creatinine (UPC) ratio was then calculated.

\section{Clinical examinations}

During each visit a complete physical examination was performed and body weight (BW) and body condition score (BCS, 1-5 point scale, [28]) were evaluated. Blood pressure (BP) measurements were also taken by an indirect Doppler method using the radial pulse with the cat in sternal recumbency or sitting. At each examination, five measures were performed and the mean value was recorded. During each visit a questionnaire was given to the owner to assess: appetite of the cat, palatability of the supplement, presence of vomit and/or diarrhoea, general health and vitality.

\section{Statistical Analysis}

The normality of the blood parameters distribution was checked by a Shapiro Wilks test for normality using the PROC UNIVARIATE. Since all distributions appeared to be non-normal $(P<0.05)$, blood parameters were compared by the Kruskall Wallis test in relation to the time of supplementation, by means of PROC NPAR1WAY. If the Kruskall Wallis test result was significant, a multiple comparison analysis based on pairwise two-sample Wilcoxon comparisons was performed $(P<0.05$ was considered significant $)$.

\section{RESULTS}

All the ten cats selected completed the study. Signalment and general characteristics are presented in Table 2. The main breed represented was European shorthair and all the animals were neutered. 9 cats were in IRIS stage 3, only 1 cat was in IRIS stage 4 .
Table 2. Signalment data of cats.

\begin{tabular}{|c|c|}
\hline Breed & \\
\hline European shorthair & $8 / 10$ \\
\hline Persian & $2 / 10$ \\
\hline \multicolumn{2}{|l|}{ Sex } \\
\hline Spayed female & $4 / 10$ \\
\hline Neutered male & $6 / 10$ \\
\hline Age (years) & $11(8.25 ; 12)$ \\
\hline \multicolumn{2}{|l|}{ IRIS stage } \\
\hline 3 & $9 / 10$ \\
\hline 4 & $1 / 10$ \\
\hline Body weight (kg) & $4(3.12 ; 5,7)$ \\
\hline $\mathrm{BCS}$ & $3(2.25 ; 3,75)$ \\
\hline \multicolumn{2}{|l|}{ Clinical signs } \\
\hline Decreased appetite & $9 / 10$ \\
\hline Vomit/diarrhea & $8 / 10$ \\
\hline
\end{tabular}

No statistical differences were recorded for ematochemical parameters measured during the study (Table 3).

Data on the biochemical profile are presented in Table 4. Crea and BUN, markers of renal function, remained stable and no significant differences between day 0 and day 60 were recorded. Statistically significant differences were only recorded for $P$ values $(P<0.0001)$ that decreased throughout the study with a reduction of $59 \%$ at T60 vs T0.

In Table 5 the hemogas parameters are presented: both $\mathrm{iCa}(P=0.0008)$ and $\mathrm{HCO} 3(P=0.0002)$ showed statistically significant differences among times of supplementation (T0, T15, T30, T60). iCa level at 60 days was the lowest and significantly different from the other levels $(P<0.05)$. There were also differences among the HCO3 levels at different times: the levels at 0 and 15 days differed $(P<0.05)$ from the levels at 30 and 60 days and it was detected a $7 \%$ increase from T0 to T60.

Urinalysis (Table 6) didn't show any significant change and UPC, UP and SG remained stable until T60. Neither for blood pressure measurements significant differences were reported (Table 7). 
All the owners reported a good palatability of the product that was consumed by all the animals during the supplementation. From T15, general health improvement was observed; at T0, $80 \%$ of the patients presented vomit and diarrhea, which were no longer reported from T15 $(P<0.05)$. Furthermore, $90 \%$ of the cats were anorexic at the first examination. Of these, $66 \%$ showed an increase in appetite during the study $(P<0.05)$. No increase of body weight or body condition score was observed.

Table 3. Ematochemical parameters measured during the study.

\begin{tabular}{|c|c|c|c|c|c|}
\hline Parameter & $\begin{array}{l}\text { Laboratory } \\
\text { standard } \\
\text { reference } \\
\text { range }\end{array}$ & T0 & $\mathrm{T} 15$ & T30 & T60 \\
\hline HТ \% & $26.00-45.00$ & $24.30(20.35 ; 31.00)$ & $25.55(21.25 ; 31.225)$ & $25.35(21.07 ; 32.87)$ & $25.35(21.57 ; 34.05)$ \\
\hline $\mathrm{HG}$ g/dl & $8.00-15.00$ & $9.95(8.97 ; 11.17)$ & $10.45(9.75 ; 11.15)$ & $10.65(10.15 ; 11.675)$ & $11.3(9.80 ; 12.45)$ \\
\hline $\mathrm{RBC} 10^{6} \mathrm{~mm}^{3}$ & $5.00-10.00$ & $5.55(5.10 ; 6.70)$ & $5.65(5.25 ; 6.65)$ & $6.05(5.62 ; 6.75)$ & $6.66(5.43 ; 6.80)$ \\
\hline WBC $10^{3} \mathrm{~mm}^{3}$ & $5.50-19.00$ & $7.25(3.85 ; 11.37)$ & $6.65(4.92 ; 14.1)$ & $8.05(5.37 ; 11.15)$ & $7.93(5.22 ; 10.27)$ \\
\hline $\mathrm{N} 10^{3} \mathrm{~mm}^{3}$ & $2.50-12.00$ & $4.15(2.34 ; 8.09)$ & $4.60(2.43 ; 9.43)$ & $4.80(3.80 ; 7.85)$ & $5.35(2.57 ; 7.57)$ \\
\hline EO $10^{3} \mathrm{~mm}^{3}$ & $0-1.50$ & $1.00(0.82 ; 1.23)$ & $1.20(1.10 ; 1.51)$ & $1.00(0.575 ; 1.245)$ & $0.95(0.72 ; 1.17)$ \\
\hline LYM $10^{3} \mathrm{~mm}^{3}$ & $1.50-7.00$ & $1.05(0.58 ; 1.97)$ & $1.25(0.66 ; 1.70)$ & $1.05(0.82 ; 1.65)$ & $1.10(1.02 ; 1.37)$ \\
\hline
\end{tabular}

Data are expressed as median (interquartile range); T: time in days.

Table 4. Biochemical parameters measured during the study.

\begin{tabular}{|c|c|c|c|c|c|}
\hline Parameter & $\begin{array}{l}\text { Laboratory } \\
\text { standard } \\
\text { reference } \\
\text { range }\end{array}$ & T0 & $\mathrm{T} 15$ & $\mathrm{~T} 30$ & T60 \\
\hline \multirow{2}{*}{ BUN mg/dL } & \multirow{2}{*}{$20.00-50.00$} & 146.50 & 154.00 & 159.00 & 150.00 \\
\hline & & $(95.50 ; 214.50)$ & $(90.25 ; 191.00)$ & $(88.50 ; 188.20)$ & $(79.05 ; 195.00)$ \\
\hline CREA mg/dL & $0.50-2.00$ & $4.00(3.50 ; 4.55)$ & $4.05(3.02 ; 4.40)$ & $3.95(3.02 ; 4.50)$ & $3.75(3.02 ; 4.42)$ \\
\hline $\mathrm{P} \mathrm{mg} / \mathrm{dL}$ & $2.70-5.00$ & $8.15(7.47 ; 8.70)$ & $5.20(4.50 ; 5.55)$ & $4.65(4.05 ; 5.02)$ & $3.35(3.13 ; 3.73)$ \\
\hline $\mathrm{TP} \mathrm{mg} / \mathrm{dL}$ & $6.00-8.00$ & $6.05(5.75 ; 6.82)$ & $6.26(5.87 ; 6.90)$ & $6.45(6.12 ; 7.03)$ & $6.60(6.17 ; 7.07)$ \\
\hline ALB mg/dL & $2.20-3.50$ & $2.75(2.40 ; 3.19)$ & $2.60(2.50 ; 3.10)$ & $3.05(2.55 ; 3.47)$ & $3.14(2.82 ; 3.37)$ \\
\hline $\mathrm{A} / \mathrm{G} \mathrm{mg} / \mathrm{dL}$ & $0.80-1.30$ & $0.65(0.52 ; 0.80)$ & $0.70(0.50 ; 0.80)$ & $0.80(0.62 ; 0.80)$ & $0.90(0.82 ; 1.00)$ \\
\hline \multirow{2}{*}{ GLU mg/dL } & \multirow{2}{*}{$60.00-120.00$} & 105.00 & 105.50 & 103.00 & 96.50 \\
\hline & & $(87.25 ; 117.75)$ & $(98.25 ; 112.00)$ & $(98.00 ; 111.25)$ & $(91.25 ; 102.75)$ \\
\hline ALT UI/L & $7.00-40.00$ & $30.50(25.00 ; 41.25)$ & $33.50(27.00 .5 ; 42)$ & $35.00(31.25 ; 39.75)$ & $35.50(31.00 ; 45.00)$ \\
\hline AST UI/L & $7.00-40.00$ & $34.50(18.50 ; 43.75)$ & $36.00(24.00 ; 45.25)$ & $32.50(18.25 ; 39.00)$ & $33.50(20.75 ; 38.00)$ \\
\hline ALP UI/L & $4.00-50.00$ & $58.50(54.00 ; 67.00)$ & $70.50(65.25 ; 78.25)$ & $61.00(54.5 ; 74.00)$ & $63.00(55.25 ; 85.25)$ \\
\hline $\mathrm{BIL} \mathrm{mg} / \mathrm{dL}$ & $0-0.50$ & $0.17(0.12 ; 0.21)$ & $0.20(0.12 ; 0.22)$ & $0.20(0.15 ; 0.24)$ & $0.15(0.14 ; 0.17)$ \\
\hline \multirow{2}{*}{ CHOL mg/dL } & \multirow{2}{*}{$70.00-150.00$} & 114.00 & 136.50 & 119.00 & 104.50 \\
\hline & & $(102.50 ; 130.00)$ & $(102.25 ; 157.50)$ & $(98.75 ; 134.00)$ & $(83.00 ; 121.00)$ \\
\hline
\end{tabular}


Table 5. Hemogas parameters measured during the study.

\begin{tabular}{cccccc}
\hline \multicolumn{7}{c}{$\begin{array}{c}\text { Laboratory } \\
\text { Parameter } \\
\text { reference } \\
\text { range }\end{array}$} & T0 & T15 & T30 & T60 \\
\hline $\mathrm{HCO} 3 \mathrm{mmol} / \mathrm{L}$ & $16.00-24.00$ & $15.70(15.70 ; 15.87)$ & $16.00(16.00 ; 16.00)$ & $16.35(16.05 ; 16.87)$ & $16.85(16.42 ; 17.00)$ \\
$\mathrm{iCa} \mathrm{mmol} / \mathrm{L}$ & $1.16-1.30$ & $1.17(1.15 ; 1.18)$ & $1.21(1.18 ; 1.22)$ & $1.22(1.19 ; 1.26)$ & $1.30(1.26 ; 1.31)$ \\
\hline
\end{tabular}

Data are expressed as median (interquartile range); T: time in days.

Table 6. Urinary parameters measured during the study.

\begin{tabular}{cccccc}
\hline \multicolumn{2}{c}{$\begin{array}{c}\text { Laboratory } \\
\text { standard } \\
\text { Parameter } \\
\text { range }\end{array}$} & T0 & T15 & T30 & T60 \\
\hline UPC & $<0.50$ & $0.55(0.40 ; 0.69)$ & $0.55(0.42 ; 0.69)$ & $0.53(0.41 ; 0.60)$ & $0.65(0.41 ; 0.74)$ \\
\hline UP mg/dL & $0-100.00$ & $362.00(218.00 ; 407.25)$ & $335.00(203.50 ; 391.25)$ & 298.50 & 236.00 \\
\hline SG & $1020-1040$ & $1015(1010 ; 1019)$ & $1017(1015 ; 1022)$ & $1018(1013 ; 1020)$ & $1025(1016 ; 1025)$ \\
\hline Data are expressed as median (interquartile range); T: time in days. & & &
\end{tabular}

Table 7. Blood pressure measured during the study.

\begin{tabular}{cccccc}
\hline Laboratory & & & & \\
Parameter & $\begin{array}{c}\text { standard } \\
\text { reference } \\
\text { range }\end{array}$ & T0 & T15 & T30 & T60 \\
\hline BP mmHg & $<95 /<150$ & $\begin{array}{c}100 / 165 \\
(90 / 148 ; 108 / 172)\end{array}$ & $\begin{array}{c}105 / 160 \\
(97 / 150 ; 112 / 170)\end{array}$ & $\begin{array}{c}105 / 160 \\
(100 / 150 ; 120 / 165)\end{array}$ & $\begin{array}{c}90 / 140 \\
(90 / 140 ; 90 / 150)\end{array}$ \\
\hline
\end{tabular}

Data are expressed as median (interquartile range); T: time in days.

\section{DISCUSSION}

Since in most CKD cases it is not possible to treat the underlying cause, treatment goals are to manage the metabolic complications in order to improve the quality of life and slow CKD progression [8].

Several markers have been associated with shorter survival in cats with CKD, including proteinuria, anaemia, and hyperphosphatemia [4]. In addition, both in humans and cats a highly significant association between increased plasma phosphate concentration and shorter survival time has been reported [20].

Phosphate is freely filtered at the glomerulus, thus its plasma concentration can be considered as a marker of the glomerular filtration rate (GFR) [4]. The mechanisms involved in phosphorus homeostasis are complicated and still not fully clear [7]. It is known that it depends on the balance between dietary intake, the exchange of phosphorus between extracellular and bone storage pools, and renal excretion [14]. It is also closely interlinked with calcium homeostasis, since both minerals plasma levels are modulated by the calciotropic hormones, PTH and calcitriol, and an increase in plasma phosphorus concentration causes a reciprocal decrease in ionized calcium concentration via the law of mass action [14].

In humans there is evidence that hyperphosphatemia is associated with vascular calcification and nephrocalcinosis, with a subsequent more rapid disease progression [19]. The situation in cats appears to be 
different. In fact, in the only published work that correlates kidney lesions with clinicopathologic variables in cats with naturally occurring CKD, plasma phosphate was associated with interstitial fibrosis but was not associated with tubular mineralisation [4]. This suggests that hyperphosphatemia in cats may not involve parenchymal mineralization but may contribute to renal fibrosis with another mechanism, thus leading to CKD progression [22]. So far this highlights the need for more aggressive control of hyperphosphatemia and renal secondary hyperparathyroidism in cats with CKD.

Therefore, the restriction of available dietary phosphorus is now recognised as one of the major contributors in slowing the disease progression and improving survival rates [2].

IRIS guidelines recommend maintaining plasma phosphate within the target reference range, which is different for each CKD stage, by reducing phosphate intake via dietary restriction, often in combination with a dietary phosphate binder.

In the present study this strategy was effective and P level decreased significantly from T0 to T60, (its median value diminishing from 8.30 to $3.58 \mathrm{mg} /$ $\mathrm{dL}$ ) and reached the target post-treatment plasma $\mathrm{P}$ concentration for IRIS stage 3 (from 2.7 to $5.0 \mathrm{mg}$ / dL) after 30 days of administration (median value at T30: $4.83 \mathrm{mg} / \mathrm{dL}$ ).

Phosphate binders are able to absorb phosphate in the intestine, forming insoluble products that are eliminated with the faeces, thus decreasing serum phosphate levels [27].

The phosphate binders in the product tested in the present trial were chitosan, calcium lactate gluconate and calcium carbonate.

Chitosan is deacetylated glucosamine made from chitin and acetyl glucosamine [23]. Previous studies in humans and cats with spontaneous CKD showed the efficacy of chitosan, alone or in combination with calcium carbonate, to control hyperphosphatemia and reduce azotemia $[2,29,30]$.

Calcium lactate gluconate is a calcium salt and it has been used as a gut phosphate binder in human medicine, in association with calcium carbonate [9]. Calcium carbonate is widely used both in human and veterinary medicine, because of its availability, ease of use and its low cost compared to other binders [16].

In human medicine it has been hypothesized that in addition to binding dietary phosphate, calcium carbonate prevents or even reverses a negative skeletal calcium balance, thus decreasing fracture risk, which is typical of CKD [16].

The use of calcium-based phosphorus binders has the recognised side effect of increasing the risk of hypercalcemia in human medicine [27], although little data is available in veterinary medicine [7], especially in cats.

In the present study, the serum ionized calcium levels of the patients were significantly higher at T60 than at $\mathrm{T} 0$, but never increased beyond the reference range (1.1-1.4 mmol/L [24]). In any case, the possible risk of hypercalcemia is the reason why it is important to check the plasma calcium concentration (preferably the ionised fraction), when monitoring a patient on a phosphate-restriction regimen using calcium-based phosphorus binders $[7,26]$.

Another important recommendation for CKD treatment is to promote alkalinisation if metabolic acidosis is present [17]. It has been reported that metabolic acidosis affects $15 \%$ of cats in IRIS Stage 3, and more than $50 \%$ of cats in IRIS Stage 4 [21].

Patient's acid-base status should therefore be checked, if possible using blood gas analysis, and on the base of the laboratory findings metabolic acidosis should be treated [25].

IRIS provides guidelines for metabolic acidosis treatment in Stage 2-4 CKD cats. If this condition is confirmed (blood bicarbonate or total $\mathrm{CO} 2<16$ $\mathrm{mmol} / \mathrm{L}$ ) sodium bicarbonate needs to be orally administered to maintain blood bicarbonate or total $\mathrm{CO} 2$ in the range of 16-24 mmol/L.

The feed supplement tested in this study also contained sodium bicarbonate.

In our study, $90 \%$ of the patients at the inclusion examination had a blood bicarbonate concentration under the normal range $(16-24 \mathrm{mmol} / \mathrm{L})$. At the end of the study, the blood bicarbonate concentration of all the patients was in the normal range, thus reaching the IRIS treatment target.

In human patients with $\mathrm{CKD}$, chronic metabolic acidosis has been associated with an alteration in bone metabolism, insulin resistance, protein-energy wasting and accelerated progression of kidney disease. A low serum bicarbonate level has also been associated with high mortality in human beings [5]. Consequently, bicarbonate supplementation has been proposed as a renoprotective strategy [1]. Evidence from clinical trials suggests that alkali therapy could retard the pro- 
gression of CKD [5]. To the authors' knowledge, no previous studies have been performed in cats to evaluate the nephroprotective property of sodium bicarbonate, and further investigations are needed.

Cats in general, and more specifically cats with $\mathrm{CKD}$, are known to have problems with their appetite [2]. It has also been reported that the addition of a phosphorus binder may reduce food intake in azotemic cats [3]. This effect was not seen in the present study, and $66 \%$ of the patients showed an increase in appetite, probably due to the improving clinical conditions.

Although the present study has yielded some findings, its design is not without flaws. They include the low number of cats in the trial and the lack of a control group. Even the duration of the clinical trial could also be considered a limitation, because CKD therapies should continue for long term periods.

\section{CONCLUSIONS}

Additional researches are required, but even with the above-mentioned limitations, we can con- clude that when a balanced diet specifically designed for cats with CKD is not sufficient to improve metabolic status of the subjects a specific supplementation is required. The supplement tested was effective in reducing blood phosphate levels and in increasing blood bicarbonate levels, thus improving the cats' clinical conditions for the duration of the study without any adverse effect.

\section{MANUFACTURERS}

${ }^{1}$ Royal Canin France. Aimargues, France.

${ }^{2}$ Istituto Candioli Farmaceutici SpA. Beinasco (TO), Italy.

${ }^{3}$ Abaxis Global Diagnostics Inc. Union City, CA, USA.

${ }^{4}$ Scil Animal Care Company S.R.L. Cassano d'Adda (MI), Italy.

${ }^{5}$ I.S.E. S.R.L. Guidonia (RM), Italy.

Ethical approval. All procedures, treatments and animal care were in compliance with the guidelines of the Italian minister of health for the care and use of animals (D.L. 4 March 2014 n. 26 and D.L. 27 January 1992 n.116) and UE (Directive 86/609/CEE).

Declaration of interest. Two of the authors are employees of Candioli Farmaceutici S.p.A.

\section{REFERENCES}

1 Adeva-Andany M.M., Fernández-Fernández C., Mouriño-Bayolo D., Castro-Quintela E. \& Domínguez-Montero A. 2014. Sodium bicarbonate therapy in patients with metabolic acidosis. The Scientific World Journal. 2014: 627-673.

2 Bernachon N., Fournel S., Gatto H., Monginoux P. \& Mcgahie D. 2014. Comparative palatability of five supplements designed for cats suffering from chronic renal disease. Irish Veterinary Journal. 67: 10-15.

3 Brown S.A., Rickertsen M. \& Sheldon S. 2008. Effects of an intestinal phosphorus binder on serum phosphorus and parathyroid hormone concentration in cats with reduced renal function. International Journal of Applied Research in Veterinary Medicine. 6: 155-160.

4 Chakrabarti S., Syme H.M. \& Elliott J. 2012. Clinicopathological variables predicting progression of azotemia in cats with chronic kidney disease. Journal of Veterinary Internal Medicine. 26: 275-281.

5 Chen W. \& Abramowitz M.K. 2014. Metabolic acidosis and the progression of chronic kidney disease. BMC Nephrology. 15: 55-62.

6 Elliott J. 2008. Retrospective analysis of dietary management of hyperphosphatemia in cats with CKD. Veterinary Focus. 18: 45-47.

7 Elliott J. 2008. Hyperphosphataemia and CKD. Veterinary Times. 38: 12-16.

8 Elliott J., Rawlings J.M., Markwell P.J. \& Barber P.J. 2000. Survival of cats with naturally occurring chronic renal failure: effect of dietary management. Journal of Small Animal Practice. 41: 235-242.

9 Emmett M. 2006. A comparison of calcium-based phosphorus binders for patients with chronic kidney disease. Dialysis \& Transplantation. 35 (5): 284-293.

10 Fédération européenne de l'industrie des aliments pour animaux familiers (FEDIAF). 2014. Nutritional Guidelines For Complete And Complementary Pet Food For Cats And Dogs. Disponível em: http://www.fediaf.org/self-regulation/ nutrition/. [Accessed December 2015].

11 Ferlizza E., Campos A., Neagu A., Cuoghi A., Bellei E., Monari E., Dondi F., Almeida A.M. \& Isani G. 2015. The effect of chronic kidney disease on the urine proteome in the domestic cat (Felis catus). Veterinary Journal. 204: 73-81.

12 Forrester S.D., Adams L.G. \& Allen T.A. 2010. Chronic Kidney Disease. In: Hand M., Thatcher C., Remillard R., Roudebush P. \& Novotny B.J. (Eds). Small Animal Clinical Nutrition. 5th edn. Topeka: Mark Morris Institute, pp.765-810. 
13 Fritsch D.A., Jewell D.E., Leventhal P.S., Brejda J., Ahle N.W., Schiefelbein H.M. \& Forrester S.D. 2015. Acceptance and effects of a therapeutic renal food in pet cats with chronic kidney disease. Veterinary Record Open. 13:2(2): e000128-e000134.

14 Geddes R.F., Finch N.C., Syme H.M. \& Elliott J. 2013. The role of phosphorus in the pathophysiology of chronic kidney disease. Journal of Veterinary Emergency and Critical Care. 23: 122-133.

15 Greene J.P., Lefebvre S.L., Wang M., Yang M., Lund E.M. \& Polzin D.J. 2014. Risk factors associated with the development of chronic kidney disease in cats evaluated at primary care veterinary hospitals. Journal of the American Veterinary Medicine Association. 244: 320-327.

16 Hill K.M., Martin B.R., Wastney M.E., McCabe G.P., Moe S.M., Weaver C.M. \& Peacock M. 2013. Oral calcium carbonate affects calcium but not phosphorus balance in stage 3-4 chronic kidney disease. Kidney International. 83(5): 959-66.

17 International Renal Interest Society (IRIS). 2013. Elanco Animal Health. Treatment Recommendations for CKD in Cats (2013). Disponível em: http://iris-kidney.com/guidelines/recommendations.aspx. [Accessed December 2015]

18 International Renal Interest Society (IRIS). 2013. Elanco Animal Health. CKD Staging in Cats (2013). Disponível em: http://iris-kidney.com/guidelines/staging.aspx. [Accessed December 2015].

19 Kalaitzidis R.G. \& Elisaf M.S. 2014. Hyperphosphatemia and phosphate binders: effectiveness and safety. Current Medical Research and Opinion. 30: 109-112.

20 King J.N., Tasker S., Gunn-Moore D.A., Strehlau G. \& the BENRIC (Benazepril In Renal Insufficiency In Cats) Study Group. 2007. Prognostic factors in cats with chronic kidney disease. Journal of Veterinary Internal Medicine. 21: 906-916.

21 Korman R.M. \& White J.D. 2013. Feline CKD: Current therapies - what is achievable? Journal of Feline Medicine and Surgery. 15(Suppl 1): 29-44.

22 Lawson J., Elliott J., Wheeler-Jones C., Syme H. \& Jepson R. 2015. Renal fibrosis in feline chronic kidney disease: known mediators and mechanisms of injury. Veterinary Journal. 203: 18-26.

23 Oh M.S. \& Uribarri J. 2014. What can we learn from the saga of chitosan gums in hyperphosphatemia therapy? Clinical Journal of the American Society of Nephrology. 9: 967-970.

24 Pettifer G. 2002. Fluids, Electrolytes, and Acid-Base Therapy. In: Slatter D.H. (Ed). Textbook of Small Animal Surgery. 3rd edn. Philadelphia: Saunders, pp.17-43.

25 Polzin D.J. 2011. Chronic kidney disease in small animals. Veterinary Clinics of North America: Small Animal Practice. 41: 15-30.

26 Polzin D.J. 2013. Evidence-based step-wise approach to managing chronic kidney disease in dogs and cats. Journal of Veterinary Emergency and Critical Care. 23: 205-215.

27 Takashima T., Sanai T., Miyazono M., Fukuda M., Kishi T., Nonaka Y., Yoshizaki M., Sato S. \& Ikeda Y. 2014. A comparison of the long-term effects of lanthanum carbonate and calcium carbonate on the course of chronic renal failure in rats with adriamycin-induced nephropathy. PLoS One 9(5): e97859.

28 Thatcher C.D., Hand M.S. \& Remillard R.L. 2010. Small Animal Clinical Nutrition: An Iterative Process. In: Hand M., Thatcher C., Remillard R., Roudebush P. \& Novotny B.J. (Eds). Small Animal Clinical Nutrition. 5th edn. Topeka: Mark Morris Institute, pp.3-21.

29 Wagner E., Schwendenwein I. \& Zentek J. 2004. Effects of a dietary chitosan and calcium supplement on Ca and P metabolism in cats. Berliner und Münchener Tierärztliche Wochenschrift. 117: 310-315.

30 Zatelli A., Pierantozzi M., D'ippolito P., Bigliati M. \& Zini E. 2012. Effect of dietary supplements in reducing probability of death for uremic crises in dogs affected by chronic kidney disease (masked RCCT). The Scientific World Journal. 2012: 219082-219088. 